\title{
The influence of neighbouring clouds on the clear sky reflectance studied with the $3-\mathrm{D}$ transport code RADUGA
}

\author{
O.V. Nikolaeva ${ }^{a}$, L.P. Bass ${ }^{\mathrm{a}}$, T.A. Germogenova ${ }^{\mathrm{a}}$, A.A. Kokhanovsky ${ }^{\text {,c, }, \text {, }}$ \\ V.S. Kuznetsov ${ }^{\mathrm{d}}$, B. Mayer ${ }^{\mathrm{e}}$ \\ ${ }^{a}$ Keldysh Institute of Applied Mathematics, Russian Academy of Sciences, Miusskaya Sq. 4, 125047 Moscow, Russia \\ ${ }^{\mathrm{b}}$ Institute of Remote Sensing, Bremen University, Otto Hahn Allee 1, 28334 Bremen, Germany \\ ${ }^{\mathrm{c}}$ Institute of Physics, National Academy of Sciences of Belarus, F. Skarina Avenue 70, 220072 Minsk, Belarus \\ 'Research Scientific Center "Kurchatov Institute", Kurchatov Sq. 1, 123182 Moscow, Russia \\ 'Institute of Atmospheric Physics, DLR, 82234 Oberpfaffenhofen, Germany
}

Received 7 May 2004; accepted 2 September 2004

\begin{abstract}
Operational remote sensing of terrestrial atmosphere is heavily based on the 1-D radiative transfer equation. However, cloudy scenes are influenced by 3-D effects (e.g., illumination from cloud sides, etc.). This leads to biases in aerosol/cloud/land/ocean retrieval schemes for scenes with clouds. These biases can be understood and quantified only with the use of the 3-D radiative transfer theory, which allows to account for arbitrary spatial variation of atmospheric parameters. The task of this paper is twofold. First of all we introduce a novel technique for the solution of the 3-D radiative transfer equation based on the grid approximations and the straightforward iteration procedure realised on supercomputers with parallel architecture. We study the performance of our technique comparing with the solutions obtained by the Monte-Carlo code. A close correspondence is found. Secondly, we quantify the influence of neighbouring clouds on the clear sky reflection function at the nadir observation depending on the solar illumination conditions. We find that the influence of cloud on the clear sky reflectance function is not negligible (even
\end{abstract}

\footnotetext{
*Corresponding author. Institute of Remote Sensing, Bremen University, Otto Hahn Allee 1, D-128334 Bremen, Germany. Tel.: + 494212184475 ; fax: +494212184555 .

E-mail address: alexk@iup.physik.uni-bremen.de (A.A. Kokhanovsky).
} 
outside the cloud geometrical shadow). Thus, the peculiar inner boundary layer arises in the sky reflectance function with shadowing and brightening effects.

(C) 2004 Elsevier Ltd. All rights reserved.

Keywords: Radiative transfer; Clouds; Atmospheric aerosol

\section{Introduction}

Radiative transfer in a cloudy atmosphere is usually studied in the framework of the planeparallel approximation. Then the diffused light field changes only along the vertical direction for a wide solar beam illumination conditions. There is no change in the radiation field in the horizontal direction. Although this approximation, which is often called 1-D case, is very important for the radiative transfer studies of extended cloudiness (e.g., extended fields of Stratocumulus clouds), it cannot be applied for majority of cloudy scenes. Indeed cloudiness has a horizontal structure (e.g., holes between clouds).

These effects can be accounted for in the framework of the 3-D radiative transfer equation, where the spatial variation of local optical properties is fully accounted for. Various approaches to deal with 3-D clouds are known [1]. Most popular techniques are Monte-Carlo method [2], the diffusion approximation [3,4], and the spherical harmonics discrete ordinate method [5-8].

In the last technique the phase function in the scattering integral is represented by the spherical harmonics and the integral is replaced by a quadrature sum. Spatial grids are introduced and obtained partial differential equations are approximated by the system of linear algebraic equations. To solve it, the successive-orders-of-scattering (SOS) approach is applied. The SOS approach has already been successfully applied in a number of cloud research topics, including the study of the transmission of thermal infrared radiation for a target-detector system [4] and the investigation of the effects of cloud geometry on the transmission of sunlight [9].

Each partial differential equation is integrated along its characteristic throughout whole calculation region in the framework of the well-known Evans's algorithm [7]. Similar methods were developed for radiation transfer calculations in nuclear reactors shielding [10,11] and for the medical physics problems [12]. These methods have some advantages and some deficiencies. In particular, some of them may be not conservative, e.g. they do not conserve the number of particles (e.g., photons, electrons, neutrons) in the transport problem at hand. This defect can lead to significant errors in the solution obtained.

Other spherical harmonics discrete ordinate methods use the local approximation for partial differential equations. Such methods were widely used in various neutron and photon transport problems in the last 50 years [3,13]. They are conservative and economic since these methods use very simple formulas and do not apply complicated logic (all spatial meshes are calculated successively). Namely, these methods have been incorporated in the RADUGA 3-D solver used in this paper $[13,14]$. They can be applied to a number of atmospheric radiative problems owing to two important features. Firstly, a special scheme for the partial differential equations solution, which obeys maximum principle, is used. Thereby, a grid solution conserves the exact solution positivity and all extrema dispositions are not corrupted by non-physical oscillations. Secondly, 
this code can deal with multiprocessor computers and, therefore, can be used to calculate photon transport in large spatial regions.

The RADUGA code can be applied to a number of atmospheric radiative problems, including studies of cloud and bright surface adjacency effects [15], cloud top topography/horizontal inhomogeneity effects [16,17], and biases in the cloud optical thickness $\tau$ retrieval from spaceborne platforms $[18,19]$, to mention a few.

In this paper the influence of a neighbouring cloud on the aerosol reflection function at the nadir observation geometry by means of the RADUGA code is studied. This is of importance for aerosol remote sensing (e.g., for a cloud screening procedures in satellite aerosol remote sensing algorithms [20]).

The paper is structured as follows. In the next section of the paper we pose a physical problem. The third section is devoted to the introduction of the 3-D radiative transport equation. A brief discussion of the numerical procedure is also given there. A detailed description of the newly developed RADUGA code used in calculations is given in separate publications [13,21]. The numerical results obtained for the simple typical aerosol-cloud model are discussed in Sections 4-6 of this work.

\section{The geometry of the problem}

The geometry of the problem is presented in Fig. 1, where a rectangular coordinate system $x y z$ is introduced. Solar light is approximated by the monodirectional source having intensity $F_{0} \delta(\mu-$ $M) \delta(\varphi-\Phi)$. Here $M=\cos \Theta, \mu=\cos \theta$ and the pair $(\Theta, \Phi)$ gives the direction of solar light propagation in the spherical coordinate system defined by the axis $z$ and angles $(\theta, \varphi)$. The azimuth $\Phi$ is counted with respect to the positive direction of the axis $x$. In this paper only results for $\Phi=0$ and $\pi$ will be reported. It means that solar light enters atmosphere from the direction of

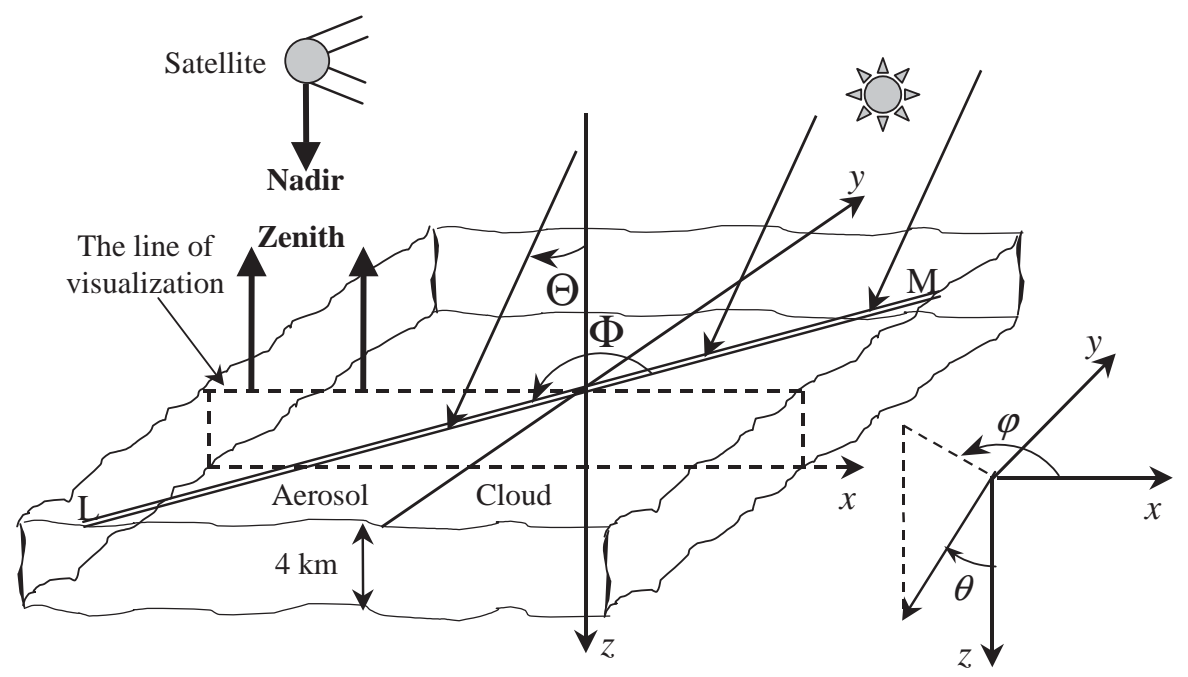

Fig. 1. The general geometry of the problem. 
the positive values of $x$ (then $\Phi=\pi$ ) or from the direction of the negative values of $x$ (then $\Phi=0$ ). The intensity of multiply scattered light is calculated along the axis $x$ in the zenith direction as shown in Fig. 1 (see the line of visualisation in Fig. 1).

We divide the terrestrial atmosphere in two equal semi-spaces separated by a local vertical plane. One part is filled by a cloudy medium and another one by the atmospheric aerosol. The processes of molecular scattering and absorption are neglected. Scattering media are assumed to be homogeneous and infinite in planes $z=$ constant. We assume that there are no light scattering particles at $z>4 \mathrm{~km}$. All downward propagated photons, which reach the plane $z=4$, are assumed to be absorbed. Therefore, the contribution of the ground albedo is neglected.

Droplets in a cloud are characterised by the Cloud C.1 particle size distribution [22] with the effective radius equal to $6 \mu \mathrm{m}$ (see Appendix). The single scattering diagram for an elementary volume of a cloudy medium is calculated at the wavelength $412 \mathrm{~nm}$ using the Mie theory [23]. The phase function in the aerosol medium is represented by the Henyey-Greenstein formula [24] (see Appendix A). The asymmetry parameter of the cloud phase function $g$ is equal to 0.85 . The value of $g$ for the aerosol phase function is equal to 0.7 . The optical thickness of cloudy and aerosol portions of the scene are varied as specified below (see Section 4). Also we have studied the variation of the reflected light as observed from a satellite for a nadir observation geometry as the function of the solar angle $\Theta$.

\section{Theory}

The radiative transfer equation is used to solve the problem. This equation has the following form for the case studied [13]:

$$
\begin{aligned}
\xi & \frac{\partial I(x, y, z, \theta, \varphi)}{\partial x}+\eta \frac{\partial I(x, y, z, \theta, \varphi)}{\partial y}+\beta \frac{\partial I(x, y, z, \theta, \varphi)}{\partial z}+\sigma_{\mathrm{ext}}(x, y, z) I(x, y, z, \theta, \varphi) \\
= & \frac{1}{4 \pi} \sigma_{\mathrm{sca}}(x, y, z) \int_{0}^{\pi} \sin \theta^{\prime} \mathrm{d} \theta^{\prime} \int_{0}^{2 \pi} I\left(x, y, z, \theta^{\prime}, \varphi^{\prime}\right) p\left(x, y, z, \chi\left(\theta, \varphi, \theta^{\prime}, \varphi^{\prime}\right)\right) \mathrm{d} \varphi^{\prime} \\
& +\frac{1}{4 \pi} \sigma_{\mathrm{sca}}(x, y, z) F_{0} p(x, y, z, \chi(\theta, \varphi, \Theta, \Phi)) \exp (-t),
\end{aligned}
$$

where $\sigma_{\text {sca }}$ and $\sigma_{\text {ext }}$ are scattering and extinction coefficients, which are assumed to be identical in this study (possible light absorption both by droplets and aerosol particles is neglected). The function $I(x, y, z, \theta, \varphi)$ is the diffused light intensity at the point $(x, y, z)$ propagated in the direction $(\theta, \varphi)$, see Fig. 1. Also we have: $\xi=\sin \theta \cos \varphi, \eta=\sin \theta \sin \varphi, \beta=\cos \theta$. The phase function $p(x, y, z, \chi)$ is defined in Appendix A. Scalar product $\chi$ is defined by the following relation:

$$
\chi\left(\theta, \varphi, \theta^{\prime}, \varphi^{\prime}\right)=\cos \theta \cos \theta^{\prime}+\sin \theta \sin \theta^{\prime} \cos \left(\varphi-\varphi^{\prime}\right) .
$$

The value of $t$ in Eq. (1) is the optical path between two points defined by radius-vectors $\vec{r}_{0}$ and $\vec{r}: t=\int_{0}^{d} \sigma_{\text {ext }}\left(\vec{r}_{0}+\zeta \vec{r}\right) \mathrm{d} \zeta$, where $d=\left|\vec{r}-\vec{r}_{0}\right|$. The vector $\vec{r}_{0}$ defines the cross point of the light beam with the boundary of scattering medium under study. 
We assume that there is no diffused light entering the medium. Therefore, boundary conditions have the following form:

$$
\begin{aligned}
& I(\vec{r}, \vec{\Omega})=0 \quad \text { at } \vec{\Omega} \vec{n}(\vec{r})<0 \text { for all } \vec{r}(x, y, z) \in G_{\text {finite }} \\
& I(\vec{r}, \vec{\Omega})=I\left(\vec{r}^{*}, \vec{\Omega}\right) \quad \text { at } \vec{\Omega} \vec{n}(\vec{r})<0 \text { for all } \vec{r}(x, y, z) \in G_{\text {infinite }}
\end{aligned}
$$

Here $G_{\text {finite }}$ is the finite part of the medium boundary (on $z$ ), $G_{\text {infinite }}$ is the infinite one (on $x$ and $y$ ), $\vec{r}^{*}$ is an inner point for the periodic boundary conditions.

We will assume that the line LM (see Fig. 1) coincides with the visualisation line. It means that the solution becomes invariant in respect to the coordinate $y$. Then we can drop the dependence on $y$ in Eq. (1) and arrive to the following simplified 2-D transport equation (see Fig. 2):

$$
\xi \frac{\partial I(x, z, \theta, \varphi)}{\partial x}+\beta \frac{\partial I(x, z, \theta, \varphi)}{\partial z}+\sigma_{\mathrm{ext}}(x, z) I(x, z, \theta, \varphi)=\hat{F} I,
$$

where

$$
\begin{aligned}
\hat{F} I \equiv & \frac{1}{4 \pi} \sigma_{\text {sca }}(x, z) \int_{0}^{\pi} \sin \theta^{\prime} \mathrm{d} \theta^{\prime} \int_{0}^{2 \pi} I\left(x, z, \theta^{\prime}, \varphi^{\prime}\right) p\left(x, z, \chi\left(\theta, \varphi, \theta^{\prime}, \varphi^{\prime}\right)\right) \mathrm{d} \varphi^{\prime} \\
& +\frac{1}{4 \pi} \sigma_{\text {sca }}(x, z) F_{0} p(x, z, \chi(\theta, \varphi, \Theta, \Phi)) \exp (-t) .
\end{aligned}
$$

Although note that the RADUGA can be applied to arbitrary-shaped scattering media.

Eq. (3) is solved using the method of successive orders of scattering. Namely, at first we neglect the integral term in Eq. (4) and calculate the diffused intensity $I(x, z, \theta, \varphi)$ from the solution of the partial differential equation. Then the obtained diffused intensity is substituted in the scattering integral in Eq. (4) and the next approximation for $I(x, z, \theta, \varphi)$ is found from the solution of the partial differential equation (3). The algorithm is stopped when the convergence is reached.

Therefore, the problem at hand is reduced to the solution of the following transport equation:

$$
\xi \frac{\partial I(x, z, \theta, \varphi)}{\partial x}+\beta \frac{\partial I(x, z, \theta, \varphi)}{\partial z}+\sigma_{\mathrm{ext}}(x) I(x, z, \theta, \varphi)=\mathfrak{\Im}(x, z, \theta, \varphi),
$$

where $I(x, z, \theta, \varphi)$ is the diffused light intensity at the point $\vec{r}(x, z)$ propagated in the direction $(\theta, \varphi), \mathfrak{I}(x, z, \theta, \varphi)=\hat{F} \tilde{I}(x, z, \theta, \varphi)$, and $\tilde{I}(x, z, \theta, \varphi)$ is the function obtained from the previous iteration as described above.

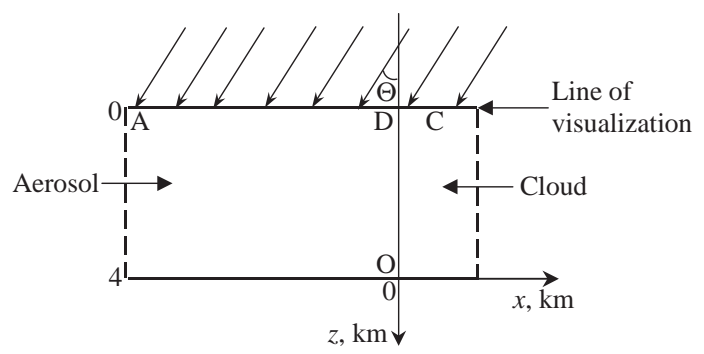

Fig. 2. The geometry of the 2-D problem. 
We introduce an angular quadrature and replace functions $I(x, z, \theta, \varphi), \tilde{I}(x, z, \theta, \varphi)$ and $\mathfrak{I}(x, z, \theta, \varphi)$ by their values in quadrature nodes. The integral $\mathfrak{I}(x, z, \theta, \varphi)$ is calculated using following standard steps:

- the expansion of the function $\tilde{I}(x, z, \theta, \varphi)$ in terms of spherical harmonics,

- the representation of the integral $\mathfrak{I}(x, z, \theta, \varphi)$ by spherical harmonics,

- the calculation of $\mathfrak{I}(x, z, \theta, \varphi)$ values in quadrature nodes.

The details will be given elsewhere [13] and will be not considered here. Variables $\theta$ and $\varphi$ are omitted for simplicity.

A standard grid method to approximate the partial differential equation of the first order (5) is used. In particular, grids with the respect to spatial variables $x$ and $z$ are introduced:

$$
x_{1 / 2}<\cdots<x_{k+1 / 2}<\cdots<x_{K+1 / 2}, \quad z_{1 / 2}<\cdots<z_{l+1 / 2}<\cdots<z_{L+1 / 2} .
$$

A single two-dimensional cell $(k, l)$ has following dimensions: $\left[x_{k-1 / 2}, x_{k+1 / 2}\right] \times\left[z_{l-1 / 2}, z_{l+1 / 2}\right]$. Correspondingly, its size is $\left[\Delta x_{k}\right] \times\left[\Delta z_{l}\right]$, where $\Delta x_{k}=x_{k+1 / 2}-x_{k-1 / 2}$ and $\Delta z_{b}=z_{b+1 / 2}-z_{b-1 / 2}$. Also the integral operator

$$
\hat{\Lambda}_{k, l}=\frac{1}{\Delta x_{k} \Delta y_{l}} \int_{x_{k-1 / 2}}^{x_{k+1 / 2}} \mathrm{~d} x \int_{z_{l-1 / 2}}^{z_{l+1 / 2}} \mathrm{~d} z
$$

is applied to both parts of Eq. (5). Then it follows:

$$
\xi\left(I_{k+1 / 2, l}-I_{k-1 / 2, l}\right) / \Delta x_{k}+\beta\left(I_{k, l+1 / 2}-I_{k, l-1 / 2}\right) / \Delta z_{l}+\sigma_{\text {ext }}^{k, l} I_{k, l}=\mathfrak{I}_{k, l},
$$

where

$$
I_{k, l}=\frac{1}{\Delta x_{k} \Delta z_{l}} \int_{x_{k-1 / 2}}^{x_{k+1 / 2}} \mathrm{~d} x \int_{z_{l-1 / 2}}^{z_{l+1 / 2}} \mathrm{~d} z I(x, z), \quad \mathfrak{I}_{k, l}=\frac{1}{\Delta x_{k} \Delta z_{l}} \int_{x_{k-1 / 2}}^{x_{k+1 / 2}} \mathrm{~d} x \int_{z_{l-1 / 2}}^{z_{l+1 / 2}} \mathrm{~d} z \mathfrak{I}(x, z)
$$

are the average values of the intensity and the source function, respectively, over a given cell and

$$
I_{k \pm 1 / 2, l}=\frac{1}{\Delta z_{l}} \int_{z_{l-1 / 2}}^{z_{l+1 / 2}} \mathrm{~d} z I\left(x_{k \pm 1 / 2}, z\right), \quad I_{k, l \pm 1 / 2}=\frac{1}{\Delta x_{k}} \int_{x_{k-1 / 2}}^{x_{k+1 / 2}} \mathrm{~d} x I\left(x, z_{l \pm 1 / 2}\right)
$$

are correspondent average values of the intensity on boundaries of the cell $(k, l)$. Fulfilment of Eq. (6) guarantees that the presented scheme is a conservative one.

Intensities $I_{k-1 / 2, l}$ and $I_{k, l-1 / 2}$ are known either from boundary conditions or from the result of the calculation for the previous cell. So we need to determine only values of $I_{k+1 / 2, l}, I_{k, l+1 / 2}$ and $I_{k, l}$. It is not possible to evaluate three parameters from a single equation (6). So we need to introduce two approximate relations among these three unknown parameters. They are given as follows:

$$
\begin{aligned}
& I_{k, l}=\left(1-v_{x, k, l}\right) I_{k+s(\xi) 2, l}+v_{x, k, l} I_{k-s(\xi) / 2, l}, \\
& I_{k, l}=\left(1-v_{z, k, l}\right) I_{k, l+s(\beta) / 2}+v_{z, k, l} I_{k, l-s(\beta) / 2},
\end{aligned}
$$


where $s(\xi)=\operatorname{sign}(\xi), s(\beta)=\operatorname{sign}(\beta), v_{x, k, l} \in[0,1)$ and $v_{z, k, l} \in[0,1)$ are weight parameters. We use values of weight parameters as introduced in [25,26]:

$$
\begin{aligned}
& v_{x, k, l}=1 /\left(2+h_{x, k, l}\right), \quad v_{z, k, l}=\frac{\left(h_{x, k, l} / h_{z, k, l}\right)\left(1+h_{x, k, l}\right)}{2+2 h_{x, k, l}+h_{x, k, l}^{2}} \quad \text { at } h_{x, k, l} \leqslant h_{z, k, l}, \\
& v_{x, k, l}=\frac{\left(h_{z, k, l} / h_{x, k, l}\right)\left(1+h_{z, k, l}\right)}{2+2 h_{z, k, l}+h_{z, k, l}^{2}}, \quad v_{z, k, l}=1 /\left(2+h_{z, k, l}\right) \quad \text { at } h_{x, k, l} \geqslant h_{z, k, l} .
\end{aligned}
$$

Optical steps $h_{x, k, l}$ and $h_{z, k, l}$ are defined as

$$
h_{x, k, l}=\sigma_{\mathrm{ext}}^{k, l} \Delta x_{k} /|\xi|, \quad h_{z, k, l}=\sigma_{\mathrm{ext}}^{k, l} \Delta z_{k} /|\beta| .
$$

This scheme defines outside fluxes $I_{k+s(\xi) / 2, l}, I_{k, l+s(\beta) / 2}$ via entering fluxes $I_{k-s(\xi) / 2, l}, I_{k, l-s(\beta) / 2}$ in a physically correct manner in any cell of any grid $[25,26]$. It permits to obtain discontinuous solutions and ones with great gradients with a high accuracy. This completes a brief description of our technique. Further details are given by Bass et al. [13,14,25,26].

\section{The validation of the RADUGA code}

\subsection{The comparison with the 1-D transport code}

Under conditions specified above, the considered problem is reduced to the 2-D problem presented in Fig. 2. Both aerosol and cloud are homogeneous along $z$-direction on the height interval $(0 \mathrm{~km}, 4 \mathrm{~km})$. They are contained in rectangular boxes. The line OD (see Fig. 2) separates aerosol medium from a cloud. Boxes are of an infinite length in the direction perpendicular to the plane of the figure of theand along axis $x$.

We will study the upwelling light field in the zenith direction at line AC. Clearly, the intensity of reflected field must depend on the coordinate $x$. This corresponds to the case of an orbiting satellite or aircraft observing aerosol-cloud system from above.

The reflection function $R$ is defined as the ratio of the light intensity $I$ reflected from a given medium to that reflected from an absolutely white Lambertian surface $I_{\mathrm{L}}$.

$$
R=I / I_{\mathrm{L}} \text {. }
$$

It is easy to show that $I_{\mathrm{L}}=\cos \Theta F_{0} / \pi$, where $F_{0}$ is the density of incident flux on the unit area perpendicular to the beam and $\Theta$ is the incident angle [24].

The largest gradients of the function $R(x)$ are expected in the area closest to the cloud boundary OD. Because both an aerosol medium and a cloud are extended to infinity along axis $x$, this function far from boundaries must be equal to the value, which can be obtained from the 1-D radiative transfer equation.

To validate our results we calculate the dependence $R(x)$ at large distances from the boundary OD using the RADUGA code and compare results obtained with values derived using an independent 1-D code based on the discrete ordinates method as described in [27]. These results are affirmed by the asymptotic theory for optically thick slabs $[28,29]$ in the case of a cloud. The 
Table 1

The comparison of the 3-D RADUGA code with 1-D code [27] results at large distances from the aerosol-cloud boundary

\begin{tabular}{lllll}
\hline & \multicolumn{2}{l}{ Aerosol $(x \rightarrow-\infty)$} & & \multicolumn{2}{l}{ Cloud $(x \rightarrow \infty)$} \\
\cline { 2 - 3 } \cline { 5 - 5 }$\Theta$ & 1-D & 3-D & 1-D & 3-D \\
\hline 50 & 0.1201 & 0.1201 & 0.6921 & 0.6925 \\
60 & 0.1494 & 0.1494 & 0.6493 & 0.6493 \\
70 & 0.1822 & 0.1823 & 0.5666 & 0.5638 \\
80 & 0.1977 & 0.1976 & 0.4476 & 0.4474 \\
\hline
\end{tabular}

comparison is shown in Table 1 assuming that the aerosol optical thickness $\tau^{\mathrm{a}}$ is equal to 1.2 and the cloud optical thickness $\tau^{\mathrm{c}}$ is equal to 30 . The solar angle is varied in the range $50-80^{\circ}$.

It follows from the analysis of Table 1 that the difference of results obtained from different codes is below $0.5 \%$ in the aerosol medium. It is smaller than $1 \%$ in the cloud. Therefore, we conclude that RADUGA is capable to reproduce 1-D code results with a high accuracy.

\subsection{The comparison of results obtained from the RADUGA code with the Monte-Carlo calculations}

We also performed extensive comparisons with the Monte-Carlo calculations based on the MYSTIC (the Monte Carlo code for the physically correct tracing of photons in cloudy atmospheres, see [30,31]). The MYSTIC is a forward Monte Carlo code which traces photons on their individual paths through the atmosphere, similar to what is described in [32]. Radiances are calculated using a local estimate technique (e.g., $[2,33])$. In this configuration, the MYSTIC has been successfully validated in the intercomparison of 3D radiation codes (see http:// climate.gsfc.nasa.gov/I3RC). The MYSTIC is operated within the libRadtran package (see http://www.libradtran.org), which prepares the optical properties of the atmosphere, to be used in the model. For the application in this paper, the atmosphere was consisted of one layer, with aerosol and cloud properties as specified in this paper. A model domain of $80 \mathrm{~km}$ in $x$ was used. A large domain size is important since the MYSTIC uses periodic boundary conditions. The model resolution was set to $0.1 \mathrm{~km}$; the MYSTIC results are, therefore, averages over $0.1 \mathrm{~km}$ bins.

Some results of comparisons are given in Figs. 3a and b (at $\Theta=60^{\circ}$ and azimuths $\Phi=0$ and $\pi$ ). We find that differences are below $1 \%$. Therefore, the RADUGA provides very accurate results as far as calculations of the light reflected by an aerosol-cloudy medium are of concern.

\section{Main properties of computed reflection functions}

Some physical dependencies are clearly seen in Figs. 3a and b. For instance, it follows from Fig. $3 b$ that there is a shadow near the cloud border for the illumination from the cloud side $(\Phi=\pi)$. Also we have a brightening effect in Fig. 3a due to the cloud side illumination effects $(\Phi=0)$. These two effects (shadowing and brightening) are primarily due to the direct light 


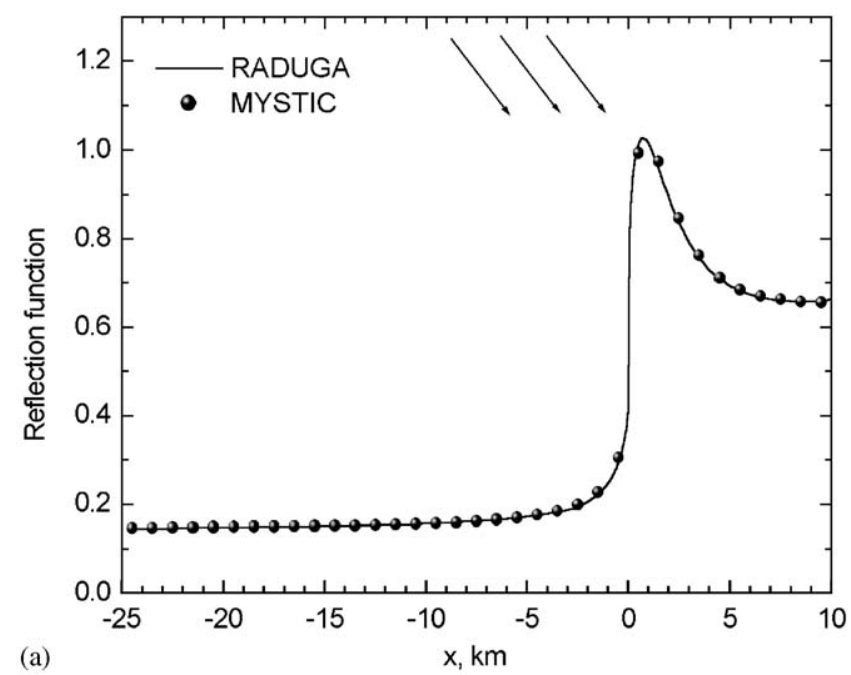

(a)

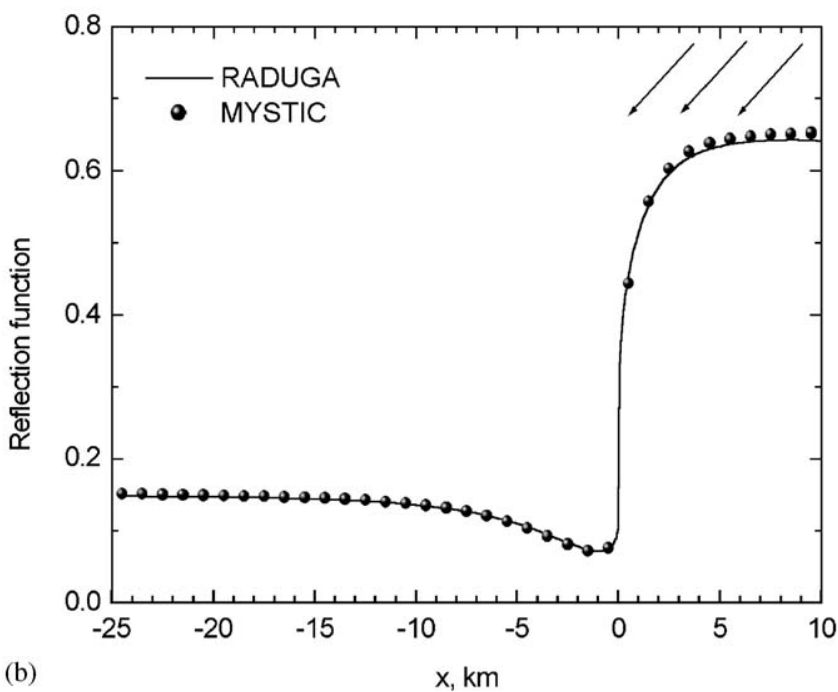

Fig. 3. The dependence $R(x)$ calculated using the Monte-Carlo code MYSTIC and the RADUGA code for the case shown in Fig. 2 at the illumination from the cloud side (a) and the aerosol side (b) for the solar angle $60^{\circ}$. The aerosol optical thickness $\tau^{\mathrm{a}}$ is equal to 1.2 and the cloud optical thickness $\tau^{\mathrm{c}}$ is equal to 30 . The phase functions are specified in the Appendix. The absorption of light and the contribution from the surface reflection is neglected. The cloud boundary is placed at $x=40 \mathrm{~km}$.

interaction with a scattering medium. They lead to roughening effects in 2-D-3-D transfer problems. We also observe (see Fig. 3b) the decrease of the reflection near the border of the cloud (inside the cloud) as compared to 1-D case. This is due to photon leaking in the area with smaller extinction coefficient in the direction of the part of the scene. The increase in the aerosol reflection function in Fig. $3 \mathrm{~b}$ close to the cloud is due to channelling of photons from a cloud to the aerosol side. These two effects (photons channelling and leaking) lead to the smoothening of the radiative 
field. Four effects considered here exist not only for a simple case studied in this paper but also for broken cloud systems [34,35].

The dependence $R(\mathrm{x})$ can be easily parameterised taking into consideration these four fundamental phenomena. The parameterisation of 3-D effects is of a great importance for satellite remote sensing because calculations presented here are computationally expensive and cannot be included in the operational aerosol/cloud retrieval algorithms [29]. Also these effects cannot be neglected. In particular, if an orbiting optical instrument observes an area correspondent to brightening/shadowing effects, then large biases in retrieved cloud/aerosol parameters are expected. This also points to the necessity of the development of the simultaneous aerosol-cloud (and surface) retrieval algorithm. The complex system should be considered in the retrieval as a whole [36].

\section{The dependence of the aerosol reflection function on the solar angle}

\subsection{The illumination from the cloud side}

1. The function $R(x)$ for the aerosol-cloudy medium specified above is shown in Fig. 4 for the case $\tau^{\mathrm{a}}=0.25, \tau^{\mathrm{c}}=30$. This dependence was obtained using the RADUGA code for solar angles $\Theta$ in the range $10-80^{\circ}, \Phi=\pi$, and the nadir observation. The characteristic feature of functions given in Fig. 4 is the presence of the brightness minimum in the aerosol area close to the cloud boundary. Because the aerosol is optically thin, the aerosol reflection function chiefly depends on the singly scattered component $R_{1}(x)$.

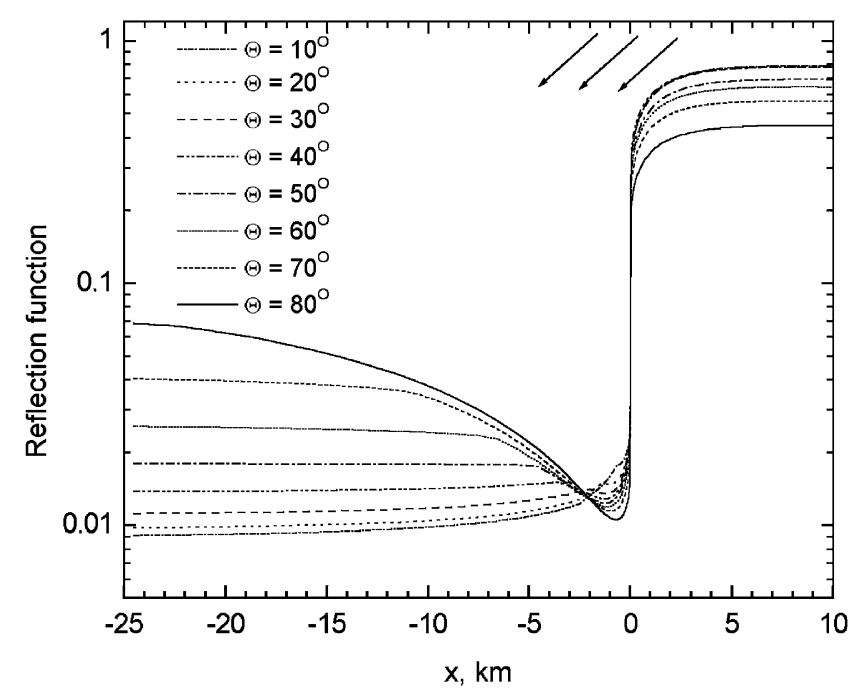

Fig. 4. The same as in Fig. 3 but for the aerosol optical thickness 0.25 and various solar angles $\left(10-80^{\circ}\right)$ at the illumination from the cloud side. 
The equation for the function $R_{1}$ can be derived from the relations (2)-(4), (7) at $\theta=\pi$, any $\varphi$, and $I\left(x, z, \theta^{\prime}, \varphi^{\prime}\right) \equiv 0$. Namely, it follows $\chi=-\cos \Theta$ and

$$
-\frac{\partial R_{1}}{\partial z}+\sigma_{\text {ext }}(x, z) R_{1}(x, z)=\frac{\sigma_{\text {ext }}(x, z)}{4 \cos \Theta} p(x, z,-\cos \Theta) \mathrm{e}^{-t(x, z)}, \quad-\infty<x<\infty, 0<z<l .
$$

Here $l=4 \mathrm{~km}$ is the cloud top height. The optical path $t(x, z)$ is determined by the following formula:

$$
t(x, z)= \begin{cases}\sigma_{\mathrm{ext}}^{\mathrm{a}} z / \cos \Theta & \text { as } z \leqslant-x \operatorname{ctg} \Theta, x<0, \\ \sigma_{\mathrm{ext}}^{\mathrm{c}} z / \cos \Theta-\left[\sigma_{\mathrm{ext}}^{\mathrm{a}}-\sigma_{\mathrm{ext}}^{\mathrm{c}}\right] x / \sin \Theta & \text { as } z \geqslant-x \operatorname{ctg} \Theta, x<0, \\ \sigma_{\mathrm{ext}}^{\mathrm{c}} z / \cos \Theta & \text { as } x \geqslant 0,\end{cases}
$$

where $\sigma_{\text {ext }}^{\mathrm{a}}$ and $\sigma_{\text {ext }}^{\mathrm{c}}$ are the extinction coefficients for the aerosol and the cloud, respectively. The boundary condition corresponds to the absence of the light propagated upwards at the border $z=l$ :

$$
R_{1}(x, l)=0 .
$$

The problem (8) has a unique solution. Namely, it follows:

$$
R_{1}(x, z)=\frac{p(x, z,-\cos \Theta)}{4 \cos \Theta} \sigma_{\text {ext }}(x, z) \int_{z}^{l} \exp \left(\sigma_{\text {ext }}(x, \zeta)(z-\zeta)\right) \exp (-t(x, \zeta)) \mathrm{d} \zeta .
$$

Substituting $z=0$, it follows:

$$
\begin{aligned}
& R_{1}(x)=u^{\mathrm{a}}(\Theta)\left[1-\mathrm{e}^{-l d^{\mathrm{a}}(\Theta) / \cos \Theta}\right] \quad \text { as } x \leqslant x_{s}, \\
& R_{1}(x)=u^{\mathrm{a}}(\Theta)\left\{1+q v^{-1}(\Theta) \mathrm{e}^{d^{\mathrm{a}}(\Theta) s(x, \Theta)}-d^{\mathrm{a}}(\Theta) v^{-1}(\Theta) \mathrm{e}^{-l v(\Theta) / \cos \Theta} \mathrm{e}^{q s(x, \Theta)}\right\} \quad \text { as } x_{s} \leqslant x<0, \\
& R_{1}(x)=u^{\mathrm{c}}(\Theta)\left[1-\mathrm{e}^{-l d^{\mathrm{c}}(\Theta) / \cos \Theta}\right] \quad \text { as } x>0 .
\end{aligned}
$$

Here

$$
\begin{aligned}
& x_{s}=-l \operatorname{tg} \Theta, \\
& s(x, \Theta)=x / \sin \Theta, \quad u^{m}(\Theta)=p^{m}(-\cos \Theta) /[4(1+\cos \Theta)], \quad q=\sigma_{\text {ext }}^{\mathrm{a}}-\sigma_{\mathrm{ext}}^{\mathrm{c}}, \\
& v(\Theta)=\sigma_{\mathrm{ext}}^{\mathrm{a}} \cos \Theta+\sigma_{\mathrm{ext}}^{\mathrm{c}}, \quad d^{m}(\Theta)=\sigma_{\mathrm{ext}}^{m}(1+\cos \Theta),
\end{aligned}
$$

where $m \equiv \mathrm{a}, \mathrm{c}$ and $p^{\mathrm{a}}$ and $p^{\mathrm{c}}$ are the aerosol and cloud phase functions, correspondingly.

The function $R_{1}(x)$ is constant as $x>0$ and as $x<x_{s}$. In interval $\left[x_{s}, 0\right)$ it is smooth and its derivative is given by following equation:

$$
(\mathrm{d} / \mathrm{d} x) R_{1}(x)=q u^{\mathrm{a}}(\Theta) v^{-1}(\Theta) d^{\mathrm{a}}(\cos \Theta) \mathrm{e}^{d^{\mathrm{a}}(\Theta) s(x, \Theta)}\left[1-\mathrm{e}^{-v(\Theta)(s(x, \Theta)+l / \cos \Theta)}\right] / \sin \Theta .
$$

Since for the considered problem the value $q$ is negative, then the component $R_{1}(x)$ monotonically decreases in this interval. The component $R_{1}(x)$ and the corresponding full solution $R(x)$ at the angle $\Theta=60^{\circ}$ are plotted in Fig. 5 .

At the media boundary (point $x=0$ ) the function $R_{1}(x)$ has a discontinuity and the value $R_{1}(0)$ is assumed to be indefinite [37]. This discontinuity of the singly scattered component $R_{1}(x)$ is due to the discontinuous optical properties of the aerosol-cloud medium under study. It is diminished 


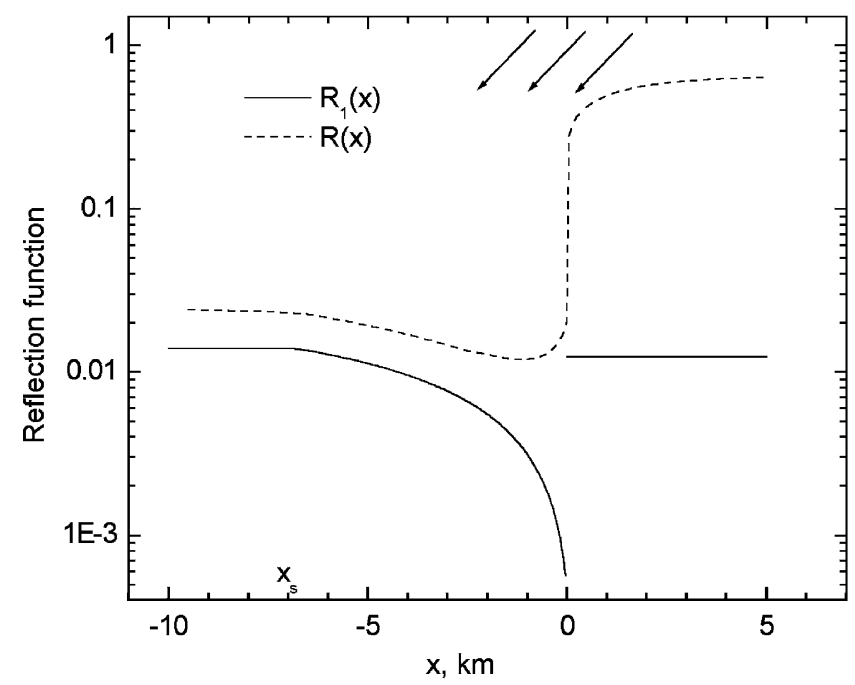

Fig. 5. Reflection function $R(x)$ and its singly scattered component for solar angle $60^{\circ}$ at the illumination from the cloud side.

by scattering acts and forms minimum of the full solution $R(x)$, look in Fig. 5. This minimum is the manifestation of the cloud shadowing effect.

2. An important point in the aerosol remote sensing from space is the estimation of the distance from the cloud, where the aerosol optical thickness can be retrieved using the 1-D transport theory. To answer on this question we present data given in Fig. 4 in yet another form.

Figs. 6a and $\mathrm{b}$ show the dependence of the relative difference $\delta$ (in percent) between the reflection function $R(x)$ obtained from the 3-D code as compared with 1-D calculations $R^{\text {a }}$ for the aerosol layer far from the cloud boundary. Namely, we define

$$
\delta(x)=1-R(x) / R^{\mathrm{a}} .
$$

Clearly, it follows as $x \rightarrow-\infty: \delta(x) \rightarrow 0$. Circles in Fig. 6b show points, where $x$ is equal to $x_{s}$. The value of $x_{s}$ gives the exact coordinate of the cloud shadow boundary for singly scattered component $R_{1}(x)$, see Eq. (10) and Fig. 5. Owing to scattering processes for the full function $R(x)$ the value $x_{s}$ is the estimate from below for the cloud shadow boundary coordinate.

We see that the functional dependence $\delta(x)$ changes its slope at $x<x_{s}$. If we tolerate the $15 \%$ error in the value of $R$, then we can state that 1-D theory can be applied at $x<x_{s}$ for the case studied here. Note that for the quantitative aerosol remote sensing values of $R$ should be correct at least within $5 \%$.

It means in particular, that the influence of cloud is of importance at distances 4.8, 6.9, 11.0, $22.7 \mathrm{~km}$ for angles equal to $50^{\circ}, 60^{\circ}, 70^{\circ}, 80^{\circ}$, respectively, for the case studied here. Clearly, no aerosol optical thickness retrievals are possible at the asymptotic case $\Theta=90^{\circ}\left(x_{s} \rightarrow-\infty\right)$. It is interesting to see that the value of $\delta$ is positive for large solar angles. This means that 2-D-3-D effects decrease the reflection function as compared to a simple 1-D theory in the case under study. Surprisingly, for sun close to the zenith the light enhancement reflectance in the clear sky 


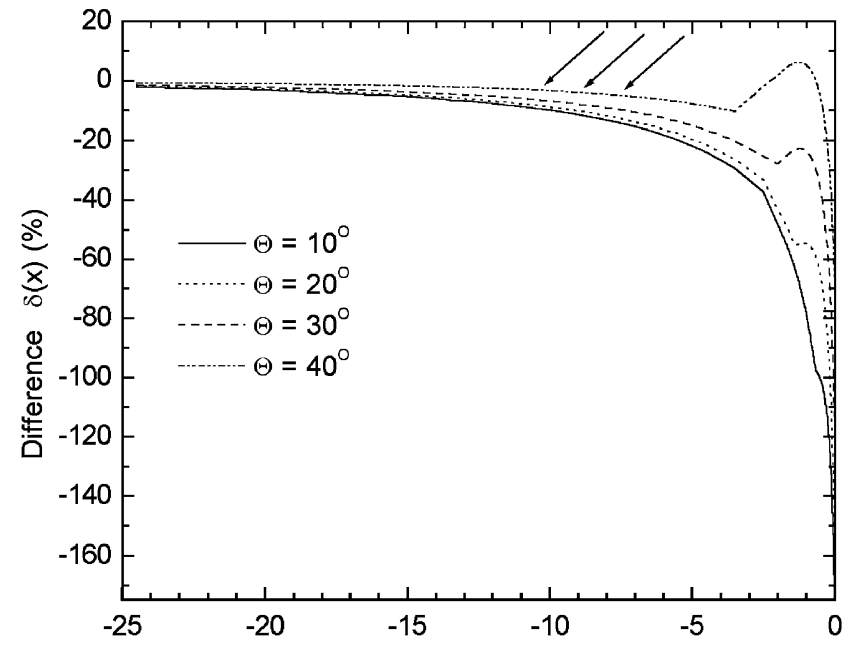

(a) $\mathrm{x}, \mathrm{km}$

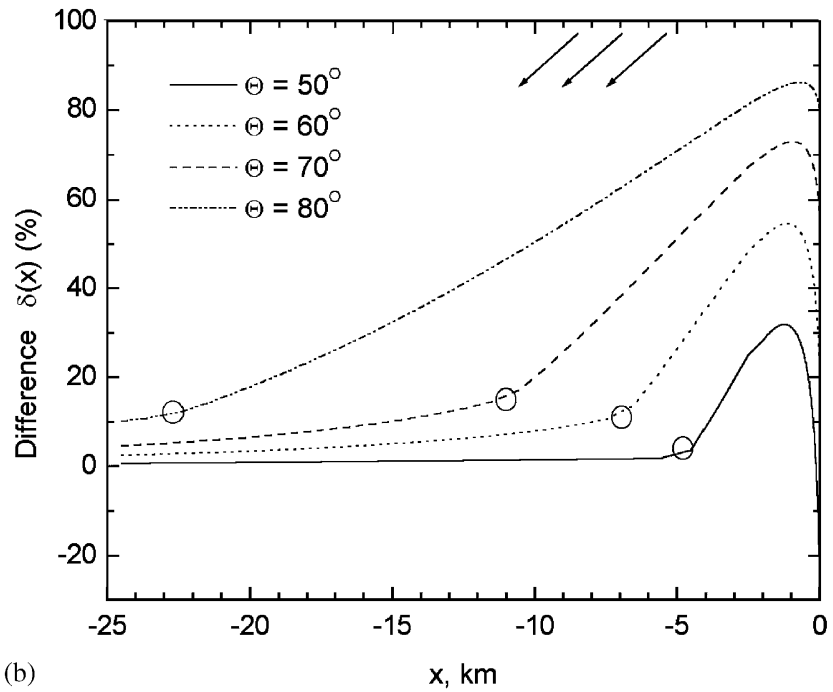

Fig. 6. The dependence $\delta(x)$ in the clear-sky part of the scene for solar angles $10-40^{\circ}$ (a) and $50-80^{\circ}$ (b) degrees correspondent to the case shown in Fig. 4.

region is possible (see Fig. 6a). For solar angles smaller than $30^{\circ}$ and for a $10 \%$ threshold the influence of cloud is of importance up to the distance $10 \mathrm{~km}$. This threshold is somewhat smaller (approximately, $5 \mathrm{~km}$ ) for solar angles $40-50^{\circ}$ but substantially increases for large solar angles (see Fig. 6b).

We also can plot the function $\delta(x)$ for a cloudy part of the scene. The dependence of the relative difference $\delta$ close to the cloud boundary is monotonous in this case (see, e.g., Fig. 7). This is primarily due to the absence of a shadowing effect for a cloud. Also we note that $\delta(x)$ is only weakly dependent on the solar angle. In particular, data presented in Fig. 7 can be approximated 


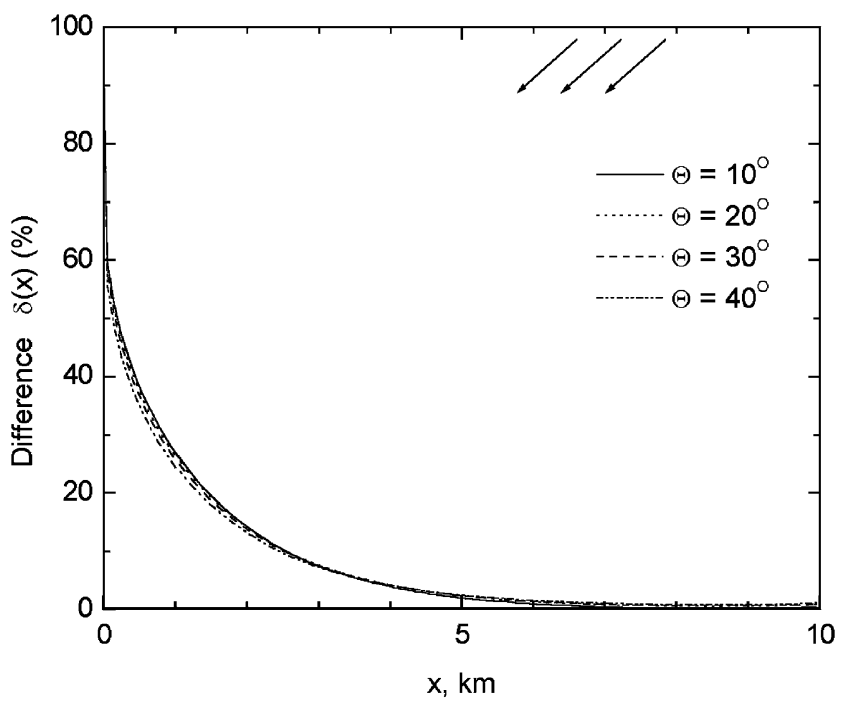

Fig. 7. The same as in Fig. 6 but for the cloudy part of the scene.

by the following curve:

$$
\delta(x)=A \exp \left(-x / x_{0}\right)
$$

where we introduced the correlation length $x_{0}$. It gives the distance, where the difference attenuates in $e$ times. We find from data given in Fig. 7 that $A=54.4$ and $x_{0}=1.4 \mathrm{~km}$. The value of $x_{0}$ should correlate with the transport length $1_{\mathrm{tr}}=1 /(1-g)$, where $1=l / \tau$ is the photon free path length in the cloud. It follows for the case we consider: $1 \approx 0.133 \mathrm{~km}^{-1}, g \approx 0.85$, and, therefore, $1_{\mathrm{tr}} \approx 0.889 \mathrm{~km}$. So we have: $x_{0} \approx 1.61_{\mathrm{tr}}$. The distance, where the difference $\delta$ is below $10 \%$ is approximately equal to $31_{\text {tr }}$ in the case studied.

3. Yet another effect is seen in the reflection function $R(x)$. Its constant value in the aerosol area far from the media boundary is more for the low sun (close to the horizon) as compared to the case of a high sun. Similar assertion is true for the singly scattered component. This follows from the expression for the derivative of the function $R_{1}(x ; \Theta)$ with respect to the parameter $\Theta$

$$
\begin{aligned}
(\mathrm{d} / \mathrm{d} \Theta) R_{1}(x ; \Theta)= & \frac{\sin \Theta}{p^{\mathrm{a}}(-\cos \Theta)}\left\{p^{\mathrm{a}}(-\cos \Theta)\left[u^{\mathrm{a}}(\Theta)-R_{1}(x)\right] l \sigma_{\text {ext }}^{\mathrm{a}} / \cos ^{2} \Theta\right. \\
& \left.+R_{1}(x)\left[(\partial / \partial \cos \Theta)\left(p^{\mathrm{a}}(-\cos \Theta)\right)+4 u^{\mathrm{a}}(\Theta)\right]\right\} \quad \text { as } x \leqslant x_{s},
\end{aligned}
$$

the positivity of Henyey-Greenstein phase function derivative and an inequity $u^{\mathrm{a}}(\Theta)-R_{1}(x) \geqslant 0$, see Eq. (9).

The opposite effect is observed for the reflection function in the cloud: its value is smaller for a high sun position as compared to the case of a low sun. This effect cannot be explained by singly scattered component analysis because the cloud is optically thick and the function $R(x)$ is formed by many scattering acts. To clarify this feature, we note that if a photon entered an optically thick non-absorbing cloud through a point of its top border placed far from the other borders, then this photon will leave the cloud through the top boundary after many collision events. The more 
collisions the photon undergoes the more its outcoming direction differs from the incident one. As a consequence, it follows that the more an incident direction differs from the axis $z$ the smaller upward reflection function value in the direction perpendicular to the cloud border.

\subsection{The illumination from the aerosol side}

The results of calculations for the illumination from the aerosol side $(\Phi=0)$ are given in Fig. 8. The sequence of curves in the aerosol/cloud side outside the transition region is similar to that given in Fig. 4 and has the same explanation. A new effect is seen however. Namely, it follows that the cloud reflection function increases close to the cloud boundary. This is due to the illumination of a cloud from a side. Therefore, the interaction of direct light with a cloud may lead not only to a familiar shadowing as in Fig. 4 at $x \approx 0$ but also to the brightening effect depending on the cloud position with respect to the incident light direction.

Such effect may be explained by the singly scattered component consideration. This function for this case is defined by relations

$$
\begin{aligned}
R_{1}(x)= & u^{\mathrm{c}}(\Theta)\left\{1-\mathrm{e}^{-l d^{\mathrm{c}}(\Theta) / \cos \Theta}\right\} \quad \text { as } x \geqslant x_{s}, \\
R_{1}(x)= & u^{\mathrm{a}}(\Theta)\left\{1-\mathrm{e}^{-l d^{\mathrm{a}}(\Theta) / \cos \Theta}\right\} \quad \text { as } x<0, \\
R_{1}(x)= & u^{\mathrm{c}}(\Theta)\left\{1-q v^{-1}(\Theta) \mathrm{e}^{-d^{\mathrm{c}}(\Theta) s(x, \Theta)}\right. \\
& \left.-d^{\mathrm{c}}(\Theta) v^{-1}(\Theta) \mathrm{e}^{-l v(\Theta) / \cos \Theta} \mathrm{e}^{q s(x, \Theta)}\right\} \quad \text { as } 0<x \leqslant x_{s} .
\end{aligned}
$$

Here $x_{s}=l \operatorname{tg} \Theta$ and other parameters are determined by the formulas (11).

In the case of illumination of cloud from the aerosol side, the dependency of the aerosol reflectance function on $x$ is a monotonous one (see Figs. 9a and b). Generally, the aerosol

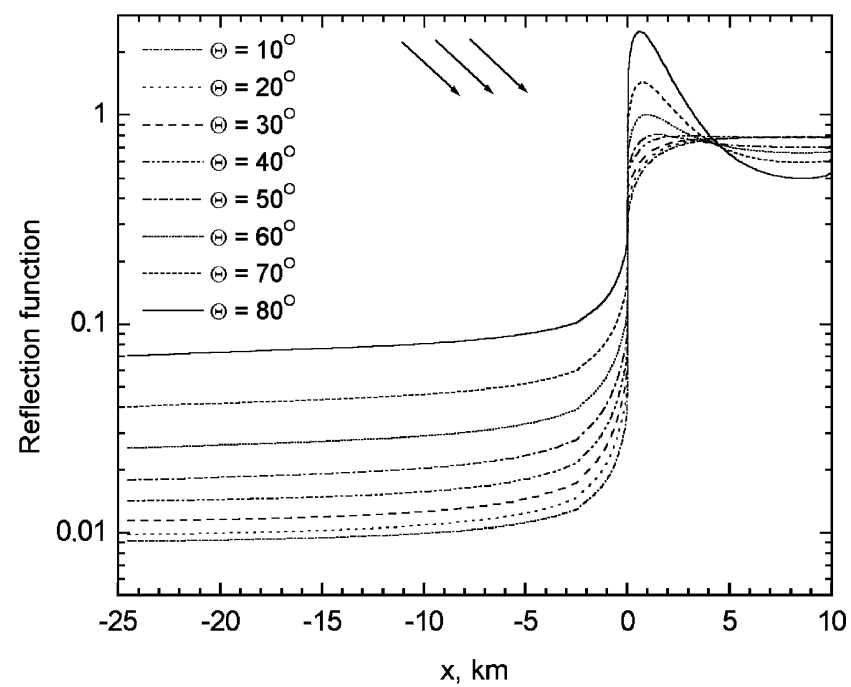

Fig. 8. The same as in Fig. 4 but the illumination from the aerosol side. 


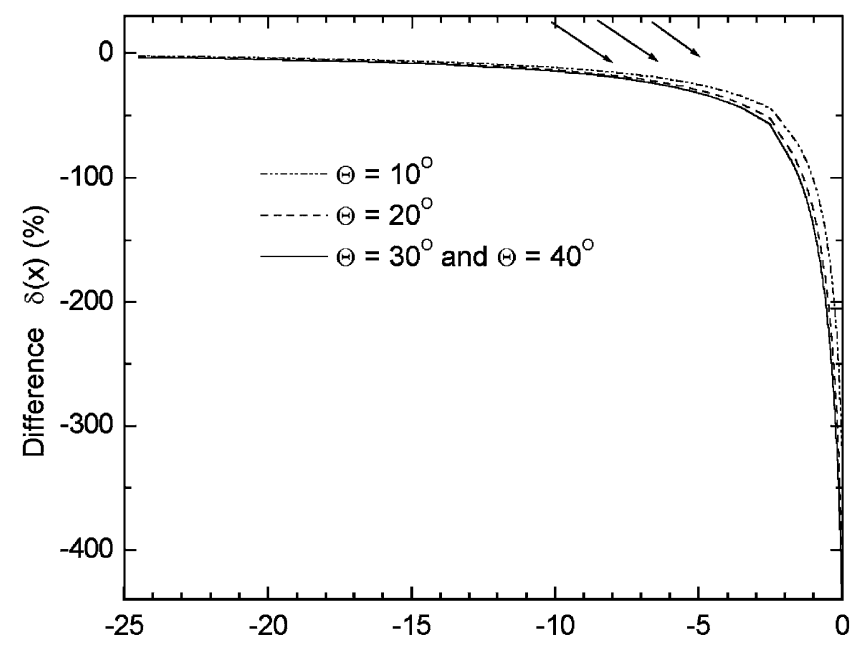

(a)

$$
\mathrm{x}, \mathrm{km}
$$

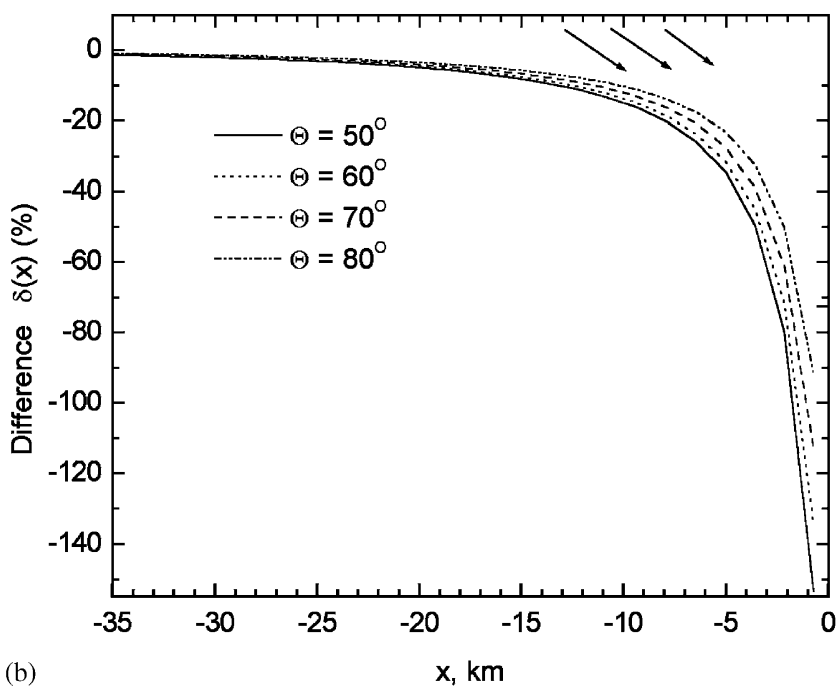

Fig. 9. The same as in Fig. 5 but for the illumination from the aerosol side.

brightness increases in the vicinity of the cloud. This increased brightness of the aerosol may lead to the positive bias in the retrieved aerosol optical thickness as derived from data obtained by space born radiometers and spectrometers. Therefore, aerosol retrievals should be performed at least at the distance $10-15 \mathrm{~km}$ from the cloud boundary (see Figs. 9a and b) depending on the illumination conditions.

Unlike the case of the illumination from the aerosol side, the cloud reflection function has a maximum, which is due to the brightening effect. The brightening effect complicates the cloud optical thickness retrieval from space. In particular, to avoid this effect only pixels positioned at $x \geqslant 2-8 \mathrm{~km}$ (depending on the solar angle, see Fig. 10) should be used in the retrieval procedure. 


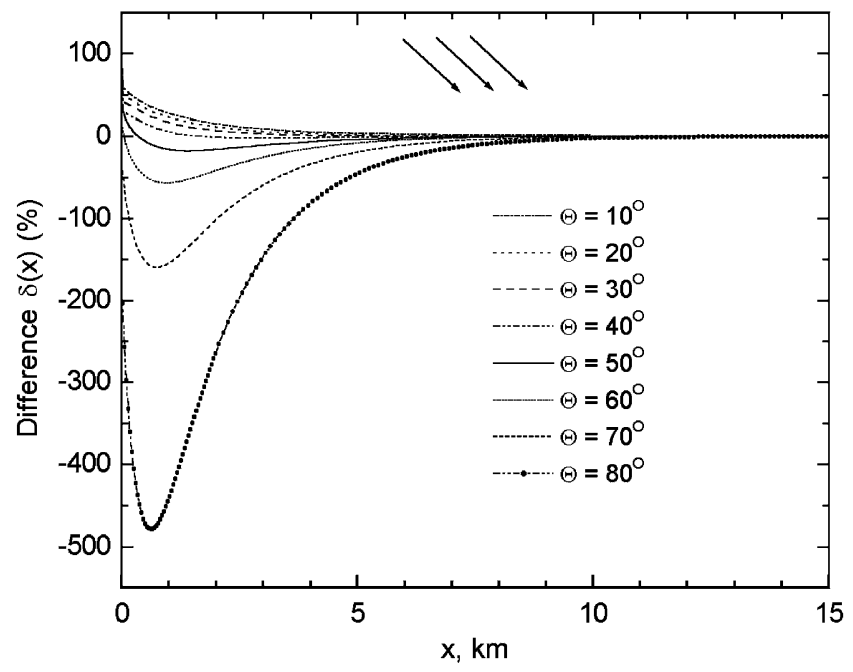

Fig. 10. The same as in Fig. 6 but for the illumination from the aerosol side.

Therefore, we see that 2-D-3-D effects influence considerably not only the aerosol reflectance but also the same is true for a cloudy part of the scene under study.

\section{Conclusions}

The developed 3-D radiative transport code RADUGA has been tested and applied to studies of the influence of cloud boundaries on the clear sky reflectance function. In the considered boundary problem for the transport equation we have investigated the phenomenon of the inner boundary layer with transient radiation regimes. The reflectance properties of this boundary layer are studied in details. We found that the aerosol reflectance is sensitive to neighbouring clouds for distances up to $25 \mathrm{~km}$ for realistic observation scenarios, depending on the solar angle. In this paper the cloud top height was fixed. Clearly, higher clouds can influence the aerosol zenith reflectance even at larger distances. Therefore, special caution should be taken in clear sky aerosol optical thickness retrieval from space even if it seems that clouds are far away from a scene studied. The same applies to the cloud optical thickness retrieval near cloud boundaries (e.g., see Fig. 10).

We conclude that 3-D effects substantially reduce the number of pixels, which can be used in the retrievals based on the 1-D radiative transfer theory. To eliminate this difficulty, retrievals should be based on the 3-D radiative transfer theory. However, this is difficult to achieve on the operational basis due to the limitations imposed by modern computers. Therefore, there is an urgent need for the parameterisation of 3-D effects in cloudy atmospheres. Such parameterisations can be easily performed for regions where radiative smoothing takes place. Corresponding dependencies $\delta(x)$ can be approximated by rather simple exponential functions (see, e.g., Eq. (12)). Parameterizations become a little bit more complex for shadowing/brightening areas due to a 
non-monotonous behaviour of studied functions there. Also the dependence on the solar angle is much more pronounced then (compare, e.g., Figs. 6 and 9).

We note that other radiative characteristics (e.g., the cloud transmittance and fluxes) can be also studied in the framework of the code developed.

\section{Acknowledgements}

This work was supported by the Russian Basic Research Foundation under grants 01-01-00570 and 02-07-90027. A.K. and B.M. acknowledges the support from the DFG Projects BU 688/8-1 and MA 2548/1-1, respectively. Discussions with J.P. Burrows are very much appreciated.

\section{Appendix A. Phase functions}

The phase function $p(\chi)$ gives the conditional probability of light scattering by an elementary volume of a scattering medium in dependence of the cosine $\chi$ of the scattering angle (the value $\chi=1$ corresponds to the forward scattering). In the transport calculations this function is represented usually by the expansion in terms of Legendre polynomials $P_{n}(\chi)$

$$
p(\chi)=\sum_{n=0}^{N}(2 n+1) g_{n} P_{n}(\chi), \quad g_{n}=\frac{1}{2} \int_{-1}^{1} p(\chi) P_{n}(\chi) \mathrm{d} \chi,
$$

where, in particular, $P_{0}(\chi)=1, P_{1}(\chi)=\chi, P_{2}(\chi)=\left(3 \chi^{2}-1\right) / 2$. The constant $g \equiv g_{1}$ is called the asymmetry parameter.

The phase function of light scattering by spherical water droplets in clouds can be calculated using the Mie theory. The dependence of the phase function on the cosine of the scattering angle $\chi$ for the Cloud C1 model [20] at the wavelength $412 \mathrm{~nm}$ is shown in Fig. 11 (left panel). Its asymmetry parameter is close to 0.85 . We use this phase function to represent light scattering by an elementary volume of a cloudy medium in this paper.
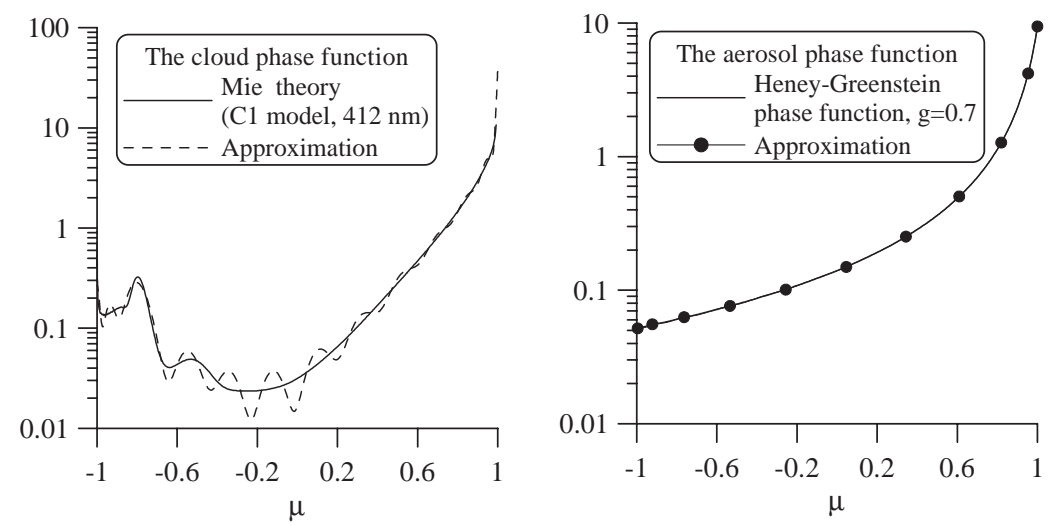

Fig. 11. The aerosol and cloud phase functions. 
Equality (A.1) for the cloud phase function has a high accuracy at $N \geqslant 350$. The number of terms $N$ influences the speed of calculations considerably. Therefore, we applied the delta- $M$ method [38] with the aim of the reduction of number of terms in the correspondent expansions. Basically, in this method the phase function is presented as a sum of a singular part (represented by a delta function) and a regular part, which can be represented by the combination of much smaller number $M$ of Legendre functions $(M \ll N)$. In this paper we have used $M=28$ (see Fig. 11). It follows that the resulted phase function (dashed line) closely corresponds to the initial Mie phase function (solid line). Note that the radiative transport in thick clouds (due to strong multiple scattering) is determined by a few first moments of the phase function (e.g., the asymmetry parameter $g$, see above). It is insured that 27 moments of the exact and approximated phase functions coincide in our case. We believe that such an approach does not influence the reflection function calculations in practical terms, which is also confirmed by comparison with other codes.

The phase function of atmospheric aerosol cannot be calculated using the Mie theory in the general case. This is due to the non-spherical shape of a great portion of aerosol particles (e.g., dust). So it is assumed that the aerosol phase function can be presented by Henyey-Greenstein formula:

$$
p(\chi)=\left(1-g^{2}\right) /\left(1+g^{2}-2 g \chi\right)^{3 / 2},
$$

here $g_{n}=g^{n}$. We choose $g=0.7$ (such value is a typical case for atmospheric aerosol) and assume in practical calculations that $N=23$. The accuracy of this approximation is shown in Fig. 11 (right panel). The error of approximation is negligible in practical terms.

Although this simplified aerosol phase function may be unrealistic as far as real atmospheric aerosol is of concern, it does not influence the main result of this work, namely the cloud adjacency effects studies. Also other researches can easily incorporate this phase function in their codes, which can facilitate the comparison of our results with those of others. We also neglect the vertical variability of the phase function as not an essential issue for the problem studied in this paper.

\section{References}

[1] Liou KN. An introduction to atmospheric radiation. New York: Academic Press; 2002.

[2] Marchuk GI, et al. The Monte-Carlo methods in atmospheric optics. Berlin: Springer; 1980.

[3] Case K, Zweifel PF. Linear transport theory. Reading, MA: Addison-Wesley; 1967.

[4] Liou KN. Radiation and cloud processes in the atmosphere. New York: Oxford University Press; 1992.

[5] Stamnes K, Thomas G. Radiative transfer in the atmosphere and ocean. Cambridge: Cambridge University Press; 1999.

[6] Chandrasekhar S. Radiative transfer. New York: Oxford University Press; 1950.

[7] Evans KF. The spherical harmonics discrete ordinate method for three-dimensional atmospheric radiative transfer. J Atmos Sci 1998;55:429-46.

[8] Lenoble J, et al. Radiative transfer in scattering and absorbing atmospheres: standard computational procedures. Hampton: A. Deepak Publishing; 1985.

[9] Liou KN, Rao N. Radiative transfer in cirrus clouds. Part IV: on cloud geometry, inhomogeneity, and absorption. J Atmos Sci 1996;53:3046-65.

[10] Postma T, Viujic J. The method of characteristics in general geometry with anisotropic scattering. Proceedings of the international conference on mathematics and computation, reactor physics and environmental analysis in nuclear application, Madrid, Spain, vol. 2. 1999. p. 1215-24. 
[11] Suslov IR. Solution of the transport equation in 2- and 3-dimensional irregular geometry by the method of characteristics. Proceedings of the international conference M\&C+SNA'93. Karlsruhe, Germany, vol. 1, 1993. p. 752.

[12] Williams ML, IIas D, Sajo D. Deterministic photon transport calculations in general geometry for external beam radiation therapy. Med Phys 2003;30:3183-95.

[13] Bass LP, et al. Discrete ordinates techniques in problems of radiative transfer. Moscow: Keldysh Institute of Applied Mathematics; 1986.

[14] Bass LP, et al. Radiative transfer universal 2D-3D-code RADUGA-5.1(P) for multiprocessor computer. EGSAGU-EUG Joint Assembly, Nice, France, vol. 1. 2003. p. 403.

[15] Kobayashi TK, et al. Monte Carlo simulations of enhanced visible radiance in clear-air satellite fields of view near clouds. J Geophys 2000;D21:26576-659.

[16] Marshak AA, et al. Radiative smoothing in fractal clouds. J Geophys Res 1995;100:26247-61.

[17] Varnai T, Marshak AA. Observations of three-dimensional radiative effects that influence MODIS cloud optical thickness retrievals. J Atmos Sci 2002;59:1607-18.

[18] Barker HW, Liu D. Inferring optical depth of broken clouds from Landsat data. J Clim 1995;8:2620-30.

[19] Loeb NG, et al. Effect of cloud inhomogeneities on the solar zenith angle dependence of nadir reflectance. J Geophys Res 1997;102:9387-95.

[20] Podgorny A. Three-dimensional radiative interactions in a polluted broken cloud system. Geophys Res Lett 2003;30 10.1029/2003GL017287.

[21] Bass LP, Nikolaeva OV, et al. Development and validation of the RADUGA-5.1(P) code. Proceedings of the workshop on algorithms and programs for neutron computations of nuclear reactors. Obninsk, Russia, 2002, to be published.

[22] Deirmendjian D. Light scattering on spherical polydispersions. Amsterdam: Elsevier; 1969.

[23] van de Hulst HC. Light scattering by small particles. New York: Wiley; 1957.

[24] van de Hulst HC. Multiple light scattering. New York: Academic Press; 1980.

[25] Bass LP, Nikolaeva OV. A positive scheme for the correct calculation of the radiation transport in strongly heterogeneous media and voids. Math Modelling 1997;9:63-72.

[26] Bass LP, Nikolaeva OV. Correct calculation of angular flux distributions in strongly heterogeneous media and voids. Proceedings of the international conference "Mathematical methods and supercomputing for nuclear applications," Saratoga Springs, USA, vol. 2, 1997. p. 995-1004.

[27] Averin AV, Voloschenko AM, et al. The ROZ-6.4 one-dimensional discrete ordinates neutrons, gamma-rays and charged particles transport code. Proceedings of the international topical meeting "Advances in mathematics, computations and reactor physics", Pittsburg, USA, vol. 5. 1991. p. 30.3.5-1-30.3.5-4.

[28] Germogenova TA. On the properties of the solution of the transport equation for a plane-parallel slab. J Comp Math Math Phys 1961;1:1001-19.

[29] Kokhanovsky AA, et al. A semi-analytical cloud retrieval algorithm using backscattered radiation in 0.4-2.4 micrometers spectral range. J Geophys Res D 2003;108:D1 10.1029/2001JD001543.

[30] Mayer B. I3RC phase 1 results from the MYSTIC Monte Carlo model. I3RC workshop. Tucson, Arizona, 1999.

[31] Mayer B. I3RC phase 2 results from the MYSTIC Monte Carlo model. I3RC workshop. Tucson, Arizona, 2000.

[32] Cahalan RF, et al. Independent pixel and Monte-Carlo estimates of stratocumulus albedo. J Atmos Sci 1994;51:3776-90.

[33] Davis JM, et al. Application of the Monte-Carlo method to problems in visibility using a local estimate: an investigation. Appl Opt 1985;24:3193-205.

[34] Faure T, et al. Neural network analysis of the radiative interaction between neighbouring pixels in inhomogeneous clouds. J Geophys Res D 2001;106:14465-84.

[35] Varnai T, Marshak AA. A method for analysing how various parts of clouds influence each other's brightness. J Geophys Res 108:10.1029/2003JD003651.

[36] Cahalan RF, et al. Cloud characterisation and clear-sky correction from Lansat-7. Remote Sensing Environ 2001;78:83-98.

[37] Germogenova TA. Local properties of transport equation solutions. Moscow: Nauka; 1985.

[38] Wiscombe WJ. The delta- $M$ method: rapid yet accurate radiative flux calculations for strongly asymmetric phase functions. J Atmos Sci 1977;34:1408-22. 